Research Journal of Applied Sciences 5 (2): 151-156, 2010

ISSN: $1815-932 \mathrm{X}$

(C) Medwell Journals, 2010

\title{
An Enhanced Packet Forwarding Scheme Using Random Way Point Mobility Model With AODV in Mobile Ad-hoc Network
}

\author{
${ }^{1} \mathrm{~S}$. Karthik, ${ }^{2} \mathrm{~A}$. Kowshika and ${ }^{3} \mathrm{C}$. Maheswari \\ Department of Computer Science and Engineering, SNS College of Technology, \\ Sathy Main Road, Coimbatore-641035, Tamil Nadu, India
}

\begin{abstract}
Discovering and maintaining routes between nodes are one of the biggest challenges in MANETs the ultimate goal of the MANET community is to provide a set of standardized protocols that can be both robust and scalable. This study proposes routing protocols based on the heading direction angle + Number of hops, Number of hops + heading direction angle, the best heading direction angle route. The first one is designed to calculate the angle direction and when the angles are the same take the best hop count. The second one is designed to calculate the best hop count and when the hops counts are the same take the best angle direction. The last one is designed to calculate the average of all heading direction angles in the route and find the best route from the source to the destination. The researchers measure the performance of the proposed approach by comparing it with the well known On-Demand (reactive) routing protocol (AODV).
\end{abstract}

Key words: Routing protocol, on dmand routing, hop count, direction angle, MANET, AODV

\section{INTRODUCTION}

A Mobile Ad-hoc Network (MANET) is a selfconfiguring network of mobile nodes connected by wireless links to form an arbitrary topology. The nodes are free to move randomly (Hu et al., 2002). Thus the network's wireless topology may be unpredictable and may change rapidly. Minimal configuration, quick deployment and absence of a central governing authority make Ad-hoc networks suitable for emergency situations like natural disasters, military conflicts, emergency medical situation, etc. Widely varying mobility characteristics are expected to have a significant impact on the performance of the routing protocols (Hsieh and Sivakumar, 2002). The overall performance of any wireless protocol depends on the duration of interconnections between any two nodes transferring data as well on the duration of interconnections between nodes of a data path containing n-nodes.

The main problem with Ad-hoc networking is how to send a message from one node to another with no direct link. The nodes in the network are moving around unpredictably and it is very challenging which nodes that are directly linked together. The topology of an Ad-hoc network is constantly changing and it is very difficult for routing process (Perkins et al., 2003; Karthik et al., 2009a). Different protocols are specialized in different aspects of routing. The mobility of the nodes affects the number of average connected paths which in turn affect the performance of the routing algorithm. With very sparsely populated network the number of possible connection between any two nodes is very less and hence, the performance is poor (Karthik et al., 2008a). It is expected that if the node density is increased the throughput of the network shall increase but beyond a certain level if density is increased the performance degrades in some protocol.

\section{MATERIALS AND METHODS}

Routing and types of routing protocols in MANET: In order to enable communication within a MANET, a routing protocol is required to establish routes between participating nodes. Because of limited transmission range, multiple network hops may be needed to enable data communication between two nodes in the network. Since MANET is an infrastructure less network, each mobile node operates not only as a host but also as a router forwarding packets for other mobile nodes in the network. There are frequent unpredictable topological changes in these networks which makes the task of finding and maintaining routes as difficult. Conventional routing protocols based on distance vector or link state algorithms cannot be applied here, since the amount of routing related traffic would waste a large portion of the wireless bandwidth (Hsieh and Sivakumar, 2002; Bai and

Corresponding Author: S. Karthik, Department of Computer Science and Engineering, SNS College of Technology, Sathy Main Road, Coimbatore-641035, Tamil Nadu, India 
Singhal, 2005) and such discovered routes would soon become obsolete due to mobility of Nodes. In MANETs mobile nodes share the same frequency channel thereby limiting the network capacity. Thus one of the highly desirable properties of a routing protocol for MANETs is that it should be bandwidth efficient. Many protocols have been proposed for MANETs. These protocols can be divided into three categories: proactive or table-driven, reactive or on-demand.

Proactive or table-driven protocols: Table-driven protocols are one of the old ways of acquiring routing in mobile Ad-hoc networks. These protocols maintain consistent overview of the network. Each node uses routing tables to store the location information of other nodes in the network. This information is used to transfer data among various nodes of the network. The freshness of the routing tables is ensured with these protocols that adopts different sorts of mechanisms. One of the adopted methods is broadcasting hello, a special message containing address information at fixed intervals of time (Hsieh and Sivakumar, 2002). On receiving this message, each node updates its routing tables with fresh locations information of other participating nodes. Destination Sequence Distance Vector routing protocol (DSDV), Wireless Routing Protocol (WRP) and Cluster-head Gateway Switch Routing (CGSR) are some of the popular table-driven protocols for mobile Ad-hoc networks. These table-driven protocols might not be considered an effective routing solution for mobile Ad-hoc network. Nodes in mobile ad-hoc networks operate with low battery power and with limited bandwidth. Presence of high mobility, large routing tables and low scalability result in consumption of bandwidth and battery life of the nodes. Moreover, continuous updates could create unnecessary network overhead.

Reactive on-demand routing protocols: Another in the family of routing protocols for mobile Ad-hoc network is on-demand routing protocols. With on-demand protocols if a source node requires a route to the destination for which it does not have route information, it initiates a route discovery process which goes from one node to the other until it reaches to the destination or an intermediate node has a route to the destination. It is the responsibility of the route request receiver node to reply back to the source node about the possible route to the destination (Karthik et al., 2008b; Hu et al., 2002). The source node uses this route for data transmission to the destination node. Some of the better known on-demand protocols are Ad-hoc On-demand Distance Vector routing (AODV), Dynamic Source Routing (DSR), Dynamic MANET
On-demand (DYMO), Location Aided Routing Protocol (LAR) and Temporary Ordered Routing Algorithm (TORA). These protocols differ on storing the previously known route information and on how they use the established route data. Again, in a network with many participating nodes the researchers may suffer with same sort of problems what they have seen in table-driven protocols.

AODV: The Ad-hoc On-demand Distance Vector (AODV) routing protocol is a reactive MANET routing protocol. Similar to DSR, AODV broadcasts a route request to discover a route in a reactive mode. The difference is that in AODV, a field of the number of hops is used in the route record, instead of a list of intermediate router addresses. Each intermediate router sets up a temporary reverse link in the process of a route discovery. This link points to the router that forwarded the request. Hence, the reply message can find its way back to the initiator when a route is discovered. When intermediate routers receive the reply, they can also set up corresponding forward routing entries. To prevent old routing information being used as a reply to the latest request, a destination sequence number is used in the route discovery packet and the route reply packet. A higher sequence number implies a more recent route request. Route maintenance in AODV is similar to that in DSR. One advantage of AODV is that AODV is loop-free due to the destination sequence numbers associated with routes.

The algorithm avoids the Bellman-Ford count to infinity problem. Therefore, it offers quick convergence when the Ad-hoc network topology changes which typically occurs when a node moves in the network. Similar to DSR, poor scalability is a disadvantage of AODV. The researchers use the example topology shown in Fig. 1 to illustrate the discovery procedure of AODV. Note that Routers $\mathrm{A}$ and $\mathrm{C}$ are disconnected from each other while both of them connect to B. When Router A starts a route discovery to $\mathrm{C}$, a route request is broadcast. The request packet contains the requested destination sequence number which is one greater than the one currently kept at A. For example, assume that the destination sequence number for $\mathrm{C}$ at $\mathrm{A}$ is $0 \times 00000000$

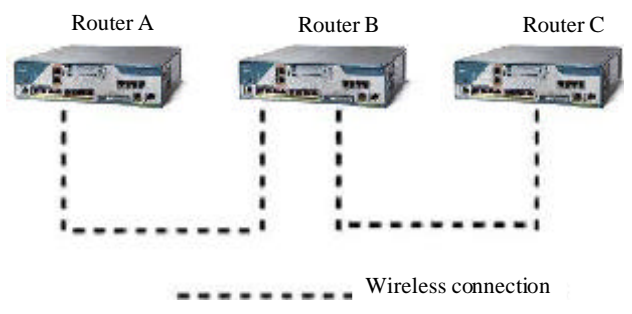

Fig. 1: Example of AODV routing protocol 
then the destination sequence number in the 20 route discovery packet is $0 \times 00000001$. The intermediate routers reply to the source if they know the route to that destination with the same or higher destination sequence number. It is assumed that $\mathrm{B}$ does not have a record for a route to $\mathrm{C}$. Therefore, $\mathrm{B}$ first sets up a temporary link pointing back to $\mathrm{A}$. In the second step, it increases the number of hops by 1 and rebroadcasts the request. When $\mathrm{C}$ receives that request, it creates a new destination sequence number. A route reply with that new sequence number is sent by $\mathrm{C}$. The initiator and all intermediate routers build routing entries associated with this new sequence number when they receive the reply. The number of hop values can be used to find a shorter path if a router receives two replies with the same destination sequence number. AODV uses a similar scheme as DSR to handle unreliable transmission of control messages.

Major MANET issue categories: In this section the MANET research issues are presented and classified. Hundreds of research aspects have been developed and discussed in this field. To analyze various research issues, this study covers most of the major investigation problems. Various fundamental and frequently discussed aspects of MANETs are identified and grouped into fifteen categories. These issues have the potential to significantly increase MANET survivability (Karthik et al., 2008c, 2009b).

Routing: Routing is an essential protocol in this field, because changes in network topology occur frequently. An efficient routing protocol is required to cope with highly fluid network conditions.

Multicasting/broadcasting: Multicast service supports users communicating with other members in a multicast group. Broadcast service supports users communicating with all members on a network.

Location service: Location information uses the Global Positioning System (GPS) or the network-based geo-location technique to obtain the physical position of a destination.

Clustering: Clustering is a method to partition the hosts into several clusters and provide a convenient framework for resource management, routing and virtual circuit support.

Mobility management: In the Ad-hoc network environment, mobile hosts can move unrestricted from place to place. Mobility management handles the storage, maintenance and retrieval of the mobile node position information.

TCP/UDP: TCP and UDP are the standard protocols used in the internet. Data applications running over MANETs such as http and real audio need transport layer protocols like TCP and UDP to send packets over the links.

IP addressing: One of the most important issues is the set of IP addresses that are assigned to the Ad-hoc network. IP addressing and address auto-configuration have attracted much attention in MANETs.

Multiple accesses: A major issue is to develop efficient medium access protocols that optimize spectral reuse and hence, maximize aggregate channel utilization in MANETs.

Radio interface: Mobile nodes rely on the radio interface or antenna to transmit packets. Packet forwarding or receiving via radio interface or antenna techniques in MANETs are useful investigations.

Bandwidth management: Bandwidth management in MANETs is a typical characterization. Because the bandwidth is usually limited, effectively managing and using it is a very important issue.

Power management: A power management approach would help reducing power consumption and hence prolonging the battery life of mobile nodes. Because most devices operate on batteries, power management becomes an important issue.

Security: The mobile nodes in MANETs are highly susceptible to malicious damage. Security issues are important in MANETs to prevent potential attacks, threats and system vulnerabilities.

Fault tolerance: This issue involves detecting and correcting faults when network failures occur. Fault-tolerance techniques are brought in for maintenance when a failure occurs during node movement, joining or leaving the network.

QoS/Multimedia: Quality of Service (QoS) and Multimedia require high bandwidth, low delay and high reliability.

Standards/products: The standards and products issues that allow the development of small scale is emerging for this field. For instance, bluetooth is a low-cost technology for short-range communications techniques. 


\section{RESULTS AND DISCUSSION}

The factors of the qualitative analysis: There are many qualitative factors related to MANETs. In this section, eight important factors are described as follows (Bai and Singhal, 2005; Wang et al., 2000).

Adaptability: It describes the reaction to change in a network. Adaptability allows the network to operate despite changes in the environment.

Flexibility: It is the ease and speed with which changes can be made to any part of the network platform and the range of changes that can be made without having to replace, redevelop or discard existing network components.

Heterogeneity: It describes a network consisting of dissimilar devices that run dissimilar protocols and in many cases support dissimilar functions or applications.

Performance: Performance is responsible for analyzing and controlling network efficiency such as network throughput and error rates.

Reliability: It was measured based on continuous error-free network operations. Perfect reliability over a given time period means no failures and no errors.

Scalability: The network must be able to grow. The initial design should grow or develop without any major changes to the overall design.

Security: Further research is needed to investigate how to stop intruders from invading the network. The network's physical facilities must be protected from harm and sensitive information kept from unauthorized users while providing simple and inexpensive access on a wide-scale basis.

Stability: In a network environment, the network topology is affected greatly by mobility. When node mobility becomes higher, the topology structure has a higher possibility for disintegration and encounters stability problems.

Algorithm: At hand are varieties of Ad-hoc routing protocols. No matter how different they may be in every routing protocol it is a key common task to find a good path between a source and a destination. Evaluation depends on a path metric such as hop count expected delay, expected lifetime, etc. As a result, the researchers

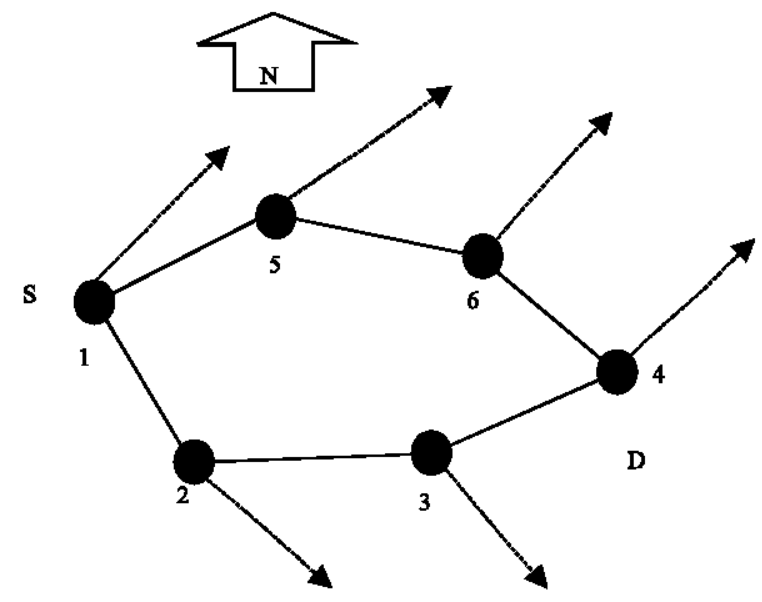

Fig. 2: Hop count and direction angle

have to find out a path that is optimal or at least nearly optimal with respect to the given or used path metric. Figure 2 shows the first new suggested metric. The source node $\mathrm{S} 1$, node 2 , node 3 and node $\mathrm{D} 4$ represent a minimum hop count route between the source $S$ and the destination $\mathrm{D}$, in normal (AODV) the protocol ignores any new route with the same or more hops in the suggested metric. If the new route has the same number of hops the protocol compares the new route angle with the old route angle and if it in a direction similar to that of the source angle the protocol will take the new route. If it is in a direction worse than the old one it will be ignored. In Fig. 2: node S1, node 5, node 6 and node D4 represent a route between the source and the destination. For forming this route, the new suggested metric is used for routing path construction (Karthik et al., 2009a,b; Hu et al., 2002). The directions of these nodes are the same or almost the same (Mauve et al., 2001). This metric gives an expectation that the connection will remain in that as long as possible. The algorithm proceeds according to the following steps:

- Calculate the heading angle for all nodes in the network

- Calculate the least hop count route between the source and the destination

- If the new route is the same number of hops compare the angles and if the new route angle is abetter than the available (current angle) update the Routing table

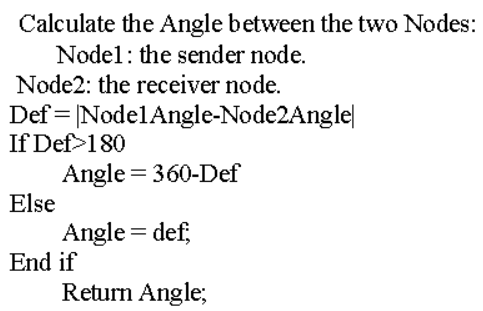




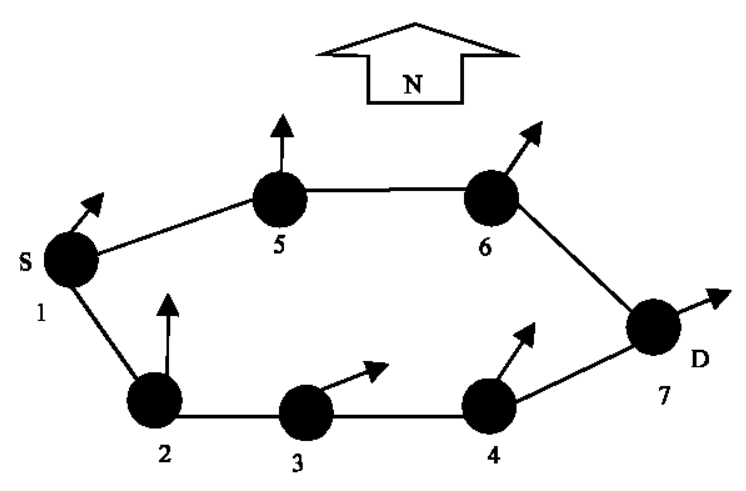

Fig. 3: Direction angle and hop count

- Check if the angle of the last node is better (Angle in the table, Angle of the node and Angle of the last node)

- Selecting the lowest node angle difference to the source node angle

Figures 3 shows the second suggested metric the source node $\mathrm{S} 1$, node 2 , node 3 , node 4 and node D7 represent the best route direction angle between the source $\mathrm{S}$ and the destination $\mathrm{D}$.

In the suggested metric, if the new route has the same angle the protocol compares the new route's number of hops with the old route's number of hops and if the new route hops are least the protocol will take the new route. If not the route is ignored (Hsieh and Sivakumar, 2002). In the Fig. 2 the new route is node S1, node 5 , node 6 and node D7. The algorithm proceeds according to the following steps.

- Calculate the heading angle for all nodes in the network

- Calculate the least direction angle route between the source and the destination

- If the new route has the same angle compare the number of hops and if the new route number of hops is a less than current route number of hops, update the routing table and take the new route. Else ignore the route

Figure 4 shows the third suggested metric if there is more than one path available calculate the average of all heading direction angles in every path and find the best average from the source to the destination. In the Fig. 4 it can be compared the paths, node $\mathrm{S} 1$, node 2 , node 3 , node 4 and node D5 and path node S1, node 6 , node 7 , node 8 , node $\mathrm{D} 5$. The algorithm proceeds according to the following steps:

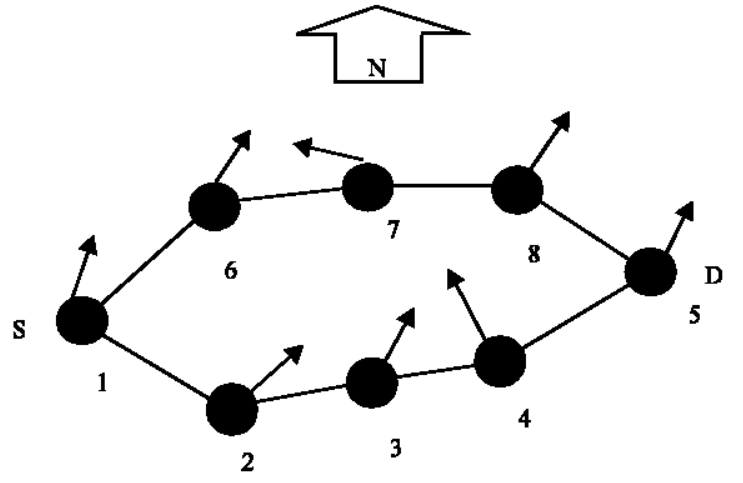

Fig. 4: Mean of all direction angles in the route

- Calculate the heading angle for all nodes in the network

- Calculate average of all heading direction angles in every path and take the best average form all routes between the source and the destination

The random waypoint model is accepted widely mainly because of its simplicity of implementation and analysis (Perkins et al., 2003; Wang et al., 2000). In this mobility model a node randomly chooses a destination and moves towards it. After reaching the destination the node stops for a time defined by the pause time parameter. After this duration, it again chooses a random destination and reports the whole process until the simulation ends.

\section{CONCLUSION}

In this study a new algorithm has been proposed which uses the heading angle and hop count along with the mean of all angle direction in its route in the Random way point mobility model. It is then compared with AODV protocol and found that this algorithm generates only a less number of broken links, smaller number of hops and only a less number of collisions compared to that of AODV protocol. As a future research, the people will use various mobility models and find an algorithm which would reduce the broken links, hop count and collisions. Along with that the researchers would continue the research adding some more additional features.

\section{ACKNOWLEDGEMENTS}

The researchers would like to thank the Director cum Secretary, Correspondent, Principal, SNS College of Technology, Coimbatore for their motivation and constant encouragement. The reseachers would like to thank the Faculty Members of Department of Computer Science and 
Engineering for critical review of this manuscript and for his valuable input and fruitful discussions. Also, he takes privilege in extending gratitude to his family members and friends who rendered their support throughout this research work.

\section{REFERENCES}

Bai, R. and M. Singhal, 2005. Supporting ETX-like metrics in AODV routing protocol. Technical Report, Department Computer Science, Univ. of Kentucky.

Hsieh, H.Y. and R. Sivakumar, 2002. On using the ad-hoc network model in wireless packet data networks. In Proceedings of the Third ACM International Symposium on Mobile Ad Hoc Networking and Computing, June 9-11, Lausanne, Switzerland, 36-47.

Hu, Y.C., D.B. Johnson and A. Perrig, 2002. SEAD: Secure efficient distance vector routing for mobile wireless Ad Hoc networks. Proceedings of the 4th IEEE Workshop on Mobile Computing Systems and Applications, June 2002, IEEE, Calicoon, New York, pp: 3-13.

Karthik, S., R.M. Bhavatharini and V.P. Arunachalam, 2008a. Analyzing interaction between denial of service (dos) attacks and threats. Proceedings of the International Conference on Computing, Communication and Networking, Dec. 18-20, IEEE Computer Society, pp: 1-9.
Karthik, S., T. Ravichandran and V.P. Arunachalam, $2008 \mathrm{~b}$. Multi directional geographical traceback with $n$ directions generalization. J. Comput. Sci., 4: 646-651.

Karthik, S., V.P. Arunachalam and T. Ravicahndran, 2008c. A comparative study of various IP trace back Strategies and simulation of IP trace back. Asian J. Inform. Technol., 7: 454-458.

Karthik. S., V.P. Arunachalam And T. Ravicahndran, 2009a. A novel direction ratio sampling algorithm (DRSA) approach for multi directional geographical traceback. Int. J. Comput. Sci. Security, 3: 272-279.

Karthik, S., T. Ravichandran and V.P. Arunachalam, $2009 \mathrm{~b}$. Analyzing interaction between denial of service (DoS) attacks and threats. Int. J. Soft Comput., 4: $68-75$.

Mauve, M. and J. Widmer and H. Hartenstein, 2001. A survey on position-based routing in mobile ad hoc networks. IEEE Network Mag., 15: 30-39.

Perkins, C., E. Belding-Royer and S. Das, 2003. Ad hoc on demand distance vector (AODV) routing. IETF Internet Draft. http://portal.acm.org/citation.cfm?id= RFC3561.

Wang, L., L. Zhang, O. Yang, Y. Shu and M. Dong, 2000. Multipath source routing in wireless ad hoc networks. Proceedings of the IEEE Canadian Conference on Electrical and Computer Engineering Halifax, May 7-10. 